\title{
Downregulation of LAPTM5 suppresses cell proliferation and viability inducing cell cycle arrest at G0/G1 phase of bladder cancer cells
}

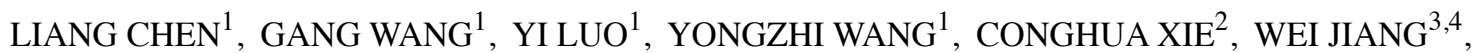 \\ YU XIAO $^{1,3}$, GUOFENG QIAN ${ }^{5}$ and XINGHUAN WANG ${ }^{1}$ \\ Departments of ${ }^{1}$ Urology, ${ }^{2}$ Radiation and Medical Oncology and ${ }^{3}$ Center for Medical Science Research, \\ Zhongnan Hospital of Wuhan University; ${ }^{4}$ Medical Research Institute, School of Medicine, Wuhan University, \\ Wuhan, Hubei 430071; ${ }^{5}$ Department of Endocrinology, The First Affiliated Hospital of Zhejiang University, \\ Hangzhou, Zhejiang 310003, P.R. China
}

Received September 26, 2016; Accepted November 24, 2016

DOI: $10.3892 /$ ijo.2016.3788

\begin{abstract}
Our transcriptome analysis revealed in bladder cancer (BCa) tissues a significant induction of lysosomalassociated multispanning membrane protein 5 (LAPTM5), a lysosomal membrane protein preferentially expressing in immune cells and hematopoietic cells. Transportation of LAPTM5 from Golgi to lysosome could be inhibited by deficiency of Nedd4, a key member of E3 ubiquitin ligase family overexpressing in invasive $\mathrm{BCa}$ and promoting its progression. Therefore, we hypothesize that LAPTM5 may be closely correlated with $\mathrm{BCa}$ tumorigenesis. In human $\mathrm{BCa}$ tissues, we observed that LAPTM5 was significantly induced at both mRNA and protein levels, which is consistent with our microarray result. Furthermore, we established a $\mathrm{BCa}$ cell model with downregulated LAPTM5, revealing a significantly delayed growth rate in the $\mathrm{BCa}$ cells with knockdown of LAPTM5. Moreover, cell cycle arrest at G0/G1 phase was triggered by decreased LAPTM5 as well, which could lead to delayed BCa cell growth. In contrast, no significant alteration of apoptosis in the BCa cells with downregulated LAPTM5 was noticed. Analysis of the changes of migration and invasion, showed significant reduced LAPTM5 suppressed cell metastasis. Furthermore, proteins involved in epithelialmesenchymal transition (EMT) were strongly altered, which
\end{abstract}

Correspondence to: Dr Guofeng Qian, Department of Endocrinology, The First Affiliated Hospital of Zhejiang University, Qingchun Road 79, Hangzhou, Zhejiang 310003, P.R. China E-mail: qgf0220@zju.edu.cn

Dr Xinghuan Wang, Department of Urology, Zhongnan Hospital of Wuhan University, Donghu Road 169, Wuhan, Hubei 430071, P.R. China

E-mail: wangxinghuan@whu.edu.cn

Key words: lysosomal-associated multispanning membrane protein 5, bladder cancer, proliferation, cell cycle arrest, migration, epithelialmesenchymal transition plays a central role in metastasis. In addition, phosphorylated ERK1/2 and p38, key members of mitogen-activated protein kinase (MAPK) family regulating BCa tumorigenesis, were strongly decreased. Taken together, our results suggested that decreased LAPTM5 inhibited proliferation and viability, as well as induced G0/G1 cell cycle arrest possibly via deactivation of ERK1/2 and p38 in BCa cells.

\section{Introduction}

Human bladder cancer (BCa) is currently one of the most common cancers worldwide (1). However, after complex therapies including surgery and antineoplastic therapy, $\mathrm{BCa}$ still frequently recurs and eventually progresses into muscleinvasive $\mathrm{BCa}(2)$. Therefore, new specific molecular markers and effective therapies are urgently needed.

Our group has collected several human BCa tissues and normal bladder tissues to conduct a microarray analysis (GEO accession no. GSE76211) $(3,4)$, revealing a significantly upregulated gene in BCa tissues, the lysosomal-associated protein multispanning transmembrane 5 (LAPTM5). LAPMT5 is a lysosomal membrane protein preferentially expressed in immune cells $(5,6)$ and hematopoietic cells $(7)$, having a close interaction with the Nedd4 (8), a member of the E3 ubiquitin ligases family (8). Nedd4 has been shown to be specifically upregulated in invasive $\mathrm{BCa}$ and be able to promote the progression of BCa (9). Moreover, some studies demonstrated that LAPTM5 was highly expressed in malignant B lymphomas and involved in B cell malignancies (10), involving in negative regulation of cell surface $\mathrm{T}$ and $\mathrm{B}$ cell receptor by promoting lysosome degradation (6). Furthermore, previous studies suggested that knockdown of LAPTM4B, another important subtype of the LAPTM family inhibited proliferation of hepatocellular carcinoma (11), prostate (12) and breast cancer cells (13).

In recent years, epithelial-mesenchymal transition (EMT) has been suggested to play a key role in the process of embryonic development, differentiation of tissues and organs, chronic inflammation and fibrosis, as well as cancer progres- 
sion (14). During EMT, cells will undergo transformation from epithelial phenotype to mesenchymal phenotype (14) and many characteristics of cells will change including loss of cell-cell adhesion and acquisition of aggressive and metastatic ability (15). Increasing evidence suggested EMT was involved in cancer invasion, metastasis (16) and the malignancy of tumors (17), often marked by reduction of E-cadherin and induction of N-cadherin (18). However, whether LAPTM5 has a connection with EMT in BCa cells remains largely unknown.

Our transcriptome analysis suggested that mitogen-activated protein kinase (MAPK) signaling pathway was linked with bladder cancer by participating in cell cycle regulation $(3,4)$. In addition, recent studies reported that LAPTM5 could diminish the activation of MAPK signaling pathway regulated by tumor necrosis factor (TNF) receptor (19). More importantly, abnormal regulation of MAPK could contribute to cancer and other human diseases $(20,21)$, including bladder cancer (3).

The exact role of LAPTM5 in tumorigenesis of human bladder cancer has not been investigated previously. In the present study, we first demonstrated that reduction of LAPTM5 had negative effects on migration, invasion and proliferation of BCa cells. Furthermore, our results suggested that alteration of MAPK signaling pathway might participate in regulation of these processes.

\section{Materials and methods}

Ethical statement for human bladder samples. As described by Cao et al and Wang et al in 2016 from our group $(3,4)$, bladder cancer and paracancerous tissue samples $(n=13)$ were obtained from patients after surgery at Zhongnan Hospital of Wuhan University, and normal bladder tissue samples $(n=3)$ were from donors by accidental death. The histology diagnosis was confirmed pathologically by two pathologists independently. All the tissues were immediately frozen and stored in liquid nitrogen or fixed in 4\% PFA after collection from the operation room. Informed consent was collected from all subjects. The study using human bladder tissue samples for RNA isolation and immunohistochemistry staining analysis was approved by the Ethics Committee at Zhongnan Hospital of Wuhan University (approval no. 2015029). All methods used for human bladder tissue samples were performed in accordance with the approved guidelines and regulations.

Human bladder cancer cell lines. The human BCa cell lines T24 (transitional cell carcinoma, cat. no. SCSP-536) and 5637 (grade II carcinoma, cat. no. TCHu 1) were obtained from Chinese Academy of Sciences in Shanghai. T24 and 5637 cell lines were identified by the China Centre for Type Culture Collection in Wuhan, China. T24 and 5637 were cultured in RPMI-1640 medium (Gibco, China) containing 10\% fetal bovine serum (FBS) (Gibco, Sydney, Australia) in a humidified atmosphere with $5 \% \mathrm{CO}_{2}$ at $37^{\circ} \mathrm{C}$.

\section{RNA expression analyses}

Total RNA isolation from bladder tissues and BCa cells. Total RNA was isolated from BCa cells and bladder tissues by RNeasy mini kit (cat. no. 74101), combined with QIAshredder (cat. no. 79654) (both from Qiagen, Hilden, Germany) using a centrifuge (cat. no. 5424; Eppendorf, Hamburg, Germany), according to the manufacturer's protocol. In order to remove genomic DNA, DNase I digestion (cat. no. 79254; Qiagen) was used in each RNA preparation.

Reverse transcription and quantitative real-time PCR ( $q R T-P C R)$. For each sample, First-Strand cDNA was synthesized using $1 \mu \mathrm{g}$ of total RNA isolated from BCa cells or bladder tissues by ReverTra Ace qPCR RT kit (Toyobo, Shanghai, China). Each reaction was conducted with $\mathrm{iQ}^{\mathrm{TM}} \mathrm{SYBR}^{\circledR}$-Green Supermix (Bio-Rad, Shanghai, China) using $1 \mu \mathrm{g}$ of cDNA in a final volume of $20 \mu \mathrm{l}$. All primers were tested for optimal annealing temperatures and PCR conditions were optimized with gradient PCRs on an iCycler (cat. no. CFX Connect; Bio-Rad, Hercules, CA, USA). Primer sequences and annealing temperatures are summarized in Table I. The cycle number of threshold (CT) value of LAPTM5 was normalized to the GAPDH value, and calculated as (22): relative gene expression $=2^{-\Delta \Delta \mathrm{ct}}, \Delta \mathrm{ct}=\mathrm{ct}_{\text {target gene }}-\mathrm{ct}_{\mathrm{GAPDH}}$, for $\mathrm{BCa}$ cells $\Delta \Delta \mathrm{ct}=\Delta \mathrm{ct}_{\text {siRNA-treated }}-\Delta \mathrm{ct}_{\text {siRNA-untreated }}$, for bladder tissues $\Delta \Delta \mathrm{ct}=\Delta \mathrm{ct}_{\mathrm{BCa}}$ tissues $-\Delta \mathrm{ct}_{\text {paracancerous tissues }}$ (ct, threshold cycle).

\section{Cell culture analyses}

Knockdown of LAPTM5 in the BCa cells. LAPTM5-target specific small interfering RNA ( $i R N A$ ) was synthesized by View Solid (Beijing, China). The sense sequence of LAPTM5target-specific-siRNA (si-LAPTM5) is as follows: siRNA1, 5'-CCACCUAUCUCAACUUCAATT-3'; siRNA2, 5'-CCAU CUACCAUGUGAUCAUTT-3'; siRNA3, 5'-GGUGCUACAG AUUGAUCAATT-3', and the sense sequence of si-control is 5'-UUCUCCGAACGUGUCAGGUTT-3'. When cells were grown to $60 \%$, T24 and 5637 cells were transfected with si-LAPTM5 and si-control using LipoJet ${ }^{\mathrm{TM}}$ (SignaGen, China). After $48 \mathrm{~h}$ transfection, alterations of LAPTM5 mRNA and protein were evaluated by qRT-PCR, immunofluorescence staining and western blot analyses.

Transwell chamber migration and invasion assay. The Transwell migration and invasion assay was conducted in 24-well plate Transwell chamber system (Corning, Inc., NY, USA). For the migration, BCa cells in serum-free medium at a density of 4-6x10 4 cells were seeded in the upper chamber (Corning, Inc.), while the lower chamber was filled with $10 \%$ FBS medium. After incubation for $24 \mathrm{~h}$ at $37^{\circ} \mathrm{C}$, the cells were removed using cotton swabs in the upper chamber. Then lower side of the chamber was fixed with 4\% PFA and stained with crystal violet, migrated cell number was counted by phase contrast microscope and statistically analyzed. To perform invasion assay, Transwell chambers were percolated with ECM Matrix gel solution (Sigma-Aldrich, St. Louis, MO, USA). Then solidified at $37^{\circ} \mathrm{C}, \sim 1 \times 10^{5}$ cells were seeded as previously described. The chamber was incubated at $37^{\circ} \mathrm{C}$ for $48 \mathrm{~h}$. The subsequent staining and observation procedures were identical to those of the migration assays.

Wound healing assay. After siRNA-transfection for $24 \mathrm{~h}$, BCa cells were scratched, and washed with PBS. Adding $0.5 \%$ FBS medium to allow cells to move into the gap, they were photographed at 0 and $12 \mathrm{~h}$ in several pre-marked spots. Migration rate was statistically analyzed using t-test.

MTT assay. After transfection for 48 h, 3,000-5,000 BCa cells $/ 200 \mu \mathrm{l}$ medium were seeded in 96 -well plates to grow for another four days. Then $20 \mu \mathrm{l}$ MTT was added in each well and 
Table I. The primers for qRT-PCR.

\begin{tabular}{|c|c|c|c|c|c|}
\hline Gene & Symbol & Forward primer $\left(5^{\prime}-3^{\prime}\right)$ & Reverse primer $\left(5^{\prime}-3^{\prime}\right)$ & $\begin{array}{c}\text { Annealing } \\
\text { temperature }\left({ }^{\circ} \mathrm{C}\right)\end{array}$ & Length (bp) \\
\hline $\begin{array}{l}\text { Lysosomal- } \\
\text { associated } \\
\text { multispanning } \\
\text { membrane } \\
\text { protein } 5\end{array}$ & LAPTM5 & $\begin{array}{l}\text { 5'-CCTGAGCCTA } \\
\text { CTGATCGGC-3' }\end{array}$ & $\begin{array}{l}\text { 5'-CAGGCACAGGA } \\
\text { GATAGTCCA-3' }\end{array}$ & 60 & 91 \\
\hline $\begin{array}{l}\text { Glyceraldehyde- } \\
\text { 3-phosphate } \\
\text { dehydrogenase }\end{array}$ & $G A P D H$ & $\begin{array}{l}\text { 5'-TGCACCACCAA } \\
\text { CTGCTTAG-3' }\end{array}$ & $\begin{array}{l}\text { 5'-GATGCAGGGA } \\
\text { TGATGTTC-3' }\end{array}$ & 60 & 176 \\
\hline
\end{tabular}

incubated at $37^{\circ} \mathrm{C}$ for $4 \mathrm{~h}$. After removing the medium, formazan precipitate was dissolved in DMSO, and absorbance at $490 \mathrm{~nm}$ was measured by a microplate reader (cat. no. SpectraMax M2; Molecular Devices, Sunnyvale, CA, USA).

Clonogenic survival assay. BCa 1,000-1,500 cells/well were seeded in new 6-well plates and grew into colonies for $\sim 15$ days. Colonies were emerged and fixed by $4 \%$ PFA for $30 \mathrm{~min}$, stained with $0.1 \%$ crystal violet for observation and photographing.

Flow cytometry analysis for cell cycle arrest and apoptosis. After harvesting and washing by PBS, BCa cells were fixed with $70 \%$ ice cold ethanol $\left(-20^{\circ} \mathrm{C}\right.$, overnight), washed again and incubated with RNaseA (20 $\mu \mathrm{g} / \mathrm{ml}$ in PBS), stained by propidium iodide $(50 \mu \mathrm{g} / \mathrm{ml})$ for $30 \mathrm{~min}$ (Sigma-Aldrich) at $37^{\circ} \mathrm{C}$ in the dark. Cell cycle were assessed on a flow cytometry (cat. no. FC500; Beckman Coulter, USA). Cell apoptosis analysis was analyzed by the flow cytometry analysis using Annexin V-fluorescence isothiocyanate (FITC)/PI apoptosis detection kit (BD Biosciences, San Jose, CA, USA), according to the manufacturer's instructions.

\section{Protein analyses}

Western blot analyses. Total protein of BCa cells was extracted using RIPA buffer containing protease inhibitor and phosphatase inhibitor (Sigma-Aldrich). Bradford protein assay (Bio-Rad, Munich, Germany) was used to measure protein concentration and Bovine serum albumin (BSA) as a standard. Protein samples were separated using 10-12.5\% SDS-PAGE and transferred to PVDF membrane (Millipore, Billerica, MA, USA). PVDF membranes were blocked in 5\% non-fat milk, then incubated with primary antibodies (Table II) and secondary antibodies (Table III). Bands were visualized and blots were exposed to Kodak Biomax MR film after using an enhanced chemiluminescence (ECL) kit (Bio-Rad).

Immunofluorescence staining for BCa cells. Coverslips were washed 3 times by cold PBS and fixed with 4\% PFA for $30 \mathrm{~min}$. Then the cells were treated by $0.1 \%$ Triton X-100 and blocked in goat serum for $30 \mathrm{~min}$, incubating with primary antibody (Table II) at room temperature for $2 \mathrm{~h}$, washing with PBS and incubating with Cy3-labeled or FITC-labeled secondary antibody (Table III) for $1 \mathrm{~h}$. Nuclei were labeled with DAPI $(2 \mu \mathrm{g} / \mathrm{ml})$. Immunofluorescence staining was analyzed using a fluorescence microscope (cat. no. IX73; Olympus, Japan).
Immunohistochemistry (IHC) staining for BCa tissue samples. Briefly, tissues were incubated with citrate buffer (0.01 M, pH 6.0) for $10 \mathrm{~min}$ after hydrated and embedded. After washing with PBS (pH 7.4) three times, tissue sections were covered with $3 \% \mathrm{H}_{2} \mathrm{O}_{2}$ for $15 \mathrm{~min}$ at room temperature and incubated with primary antibody overnight at $4^{\circ} \mathrm{C}$. After a washing procedure, biotinylated secondary antibody was incubated with the section for $30 \mathrm{~min}$. Then DAB substrate chromogen solution was added before tissue sections were incubated with HRP substrate solution for $30 \mathrm{~min}$. Slides were counterstained for $1 \mathrm{~min}$ with hematoxylin, then dehydrated and analyzed by microscopy.

Statistical analyses. All analyses were performed three times and represent data from three individual experiments. Two-tailed Student's t-test was used for significance of differences between subgroups. Statistical analyses were performed with SPSS 16.0. Statistical significance was set at probability values of $\mathrm{p}<0.05$

\section{Results}

Upregulation of LAPTM5 in BCa tissues compared with paracancerous tissues and normal bladder tissues. Oncomine database (www.oncomine.org) showed that LAPTM5 was significantly upregulated at the transcriptional level in $\mathrm{BCa}$ tissues compared with normal bladder tissues (Fig. 1A), which is consistent with our microarray data. Furthermore, LAPTM5 also exhibited a significant upregulation in the $\mathrm{BCa}$ tissues compared with the paired paracancerous tissues $(n=13)$ (Fig. 1B). In addition, immunohistochemistry staining revealed strong increase of LAPTM5 protein in the BCa tissues, compared with paracancerous bladder tissues (Fig. 1C).

Knockdown of LAPTM5 significantly inhibits the transcription and protein levels of LAPTM5. To construct a cell model of LAPTM5 deficiency, we used three distinct LAPTM5-target-specific-siRNA to transfect T24 and 5637. After $48 \mathrm{~h}$, the knockdown efficiency was validated by qRT-PCR (Fig. 2A and B) and western blot analysis (Fig. 2C). Moreover, immunofluorescence staining also showed the abundance of LAPTM5 protein was strongly downregulated (Fig. 2D). The result showed that LAPTM5 expression at both transcriptional and translational levels was significantly reduced with LAPTM5-target-specific-siRNA in the BCa cells. 
Table II. The primary antibodies.

\begin{tabular}{|c|c|c|c|c|}
\hline Antigens & $\begin{array}{l}\text { Species antibodies } \\
\text { raised in }\end{array}$ & $\begin{array}{l}\text { Dilution } \\
\text { (IF) }\end{array}$ & $\begin{array}{l}\text { Dilution } \\
\text { (WB) }\end{array}$ & Supplier \\
\hline E-Cadherin, human & Rabbit, monoclonal & $1: 200$ & $1: 500$ & $\begin{array}{l}\text { Cell Signaling Technology, USA, } \\
\text { cat. no. } 3195\end{array}$ \\
\hline N-Cadhern, human & Rabbit, monoclonal & $1: 200$ & $1: 1,000$ & $\begin{array}{l}\text { Cell Signaling Technology, USA, } \\
\text { cat. no. } 13116\end{array}$ \\
\hline$\beta$-Catenin, human & Rabbit, monoclonal & - & $1: 1,000$ & $\begin{array}{l}\text { Cell Signaling Technology, USA, } \\
\text { cat. no. } 8480\end{array}$ \\
\hline Ki-67, human & Rabbit, monoclonal & $1: 200$ & - & $\begin{array}{l}\text { Novus Biologicals, USA, } \\
\text { cat. no. NBP2-19012 }\end{array}$ \\
\hline Slug, human & Rabbit, monoclonal & - & $1: 1,000$ & $\begin{array}{l}\text { Cell Signaling Technology, USA, } \\
\text { cat. no. } 9585\end{array}$ \\
\hline Claudin-1, human & Rabbit, monoclonal & - & $1: 10,000$ & $\begin{array}{l}\text { Cell Signaling Technology, USA, } \\
\text { cat. no. } 13255\end{array}$ \\
\hline $\begin{array}{l}\text { Glyceraldehyde 3-phosphate } \\
\text { dehydrogenase (GAPDH), human }\end{array}$ & Mouse, monoclonal & - & $1: 2,000$ & $\begin{array}{l}\text { Santa Cruz Biotechnology Inc., USA, } \\
\text { cat. no. sc- } 365062\end{array}$ \\
\hline Cyclin D1, human & Rabbit, monoclonal & - & $1: 1,000$ & $\begin{array}{l}\text { Cell Signaling Technology, USA, } \\
\text { cat. no. } 2978\end{array}$ \\
\hline CDK2, human & Rabbit, monoclonal & - & $1: 1,000$ & $\begin{array}{l}\text { Cell Signaling Technology, USA, } \\
\text { cat. no. } 2546\end{array}$ \\
\hline CDK4, human & Rabbit, monoclonal & - & $1: 1,000$ & Abcam, UK, cat. no. ab108357 \\
\hline Cyclin A1/A2 & Rabbit, monoclonal & - & $1: 1,000$ & Abcam, UK, cat. no. ab185619 \\
\hline p-GSK3 $\beta$, human & Rabbit, monoclonal & - & $1: 10,000$ & $\begin{array}{l}\text { Cell Signaling Technology, USA, } \\
\text { cat. no. } 5558 \mathrm{~S}\end{array}$ \\
\hline GSK $3 \beta$, human & Rabbit, monoclonal & - & $1: 10,000$ & $\begin{array}{l}\text { Cell Signaling Technology, USA, } \\
\text { cat. no. } 12456 \mathrm{~S}\end{array}$ \\
\hline LAPTM5, human & Rabbit, monoclonal & $1: 50$ & $1: 1,000$ & Abcam, UK, cat. no. ab108014 \\
\hline $\begin{array}{l}\text { Phospho-p44/42 MAPK (Erk 1/2) } \\
\text { (Thr202/Tyr204), human }\end{array}$ & Rabbit, monoclonal & - & $1: 1,000$ & $\begin{array}{l}\text { Cell Signaling Technology, USA, } \\
\text { cat. no. } 4370\end{array}$ \\
\hline p44/42 MAPK (Erk1/2), rat & Rabbit, monoclonal & - & $1: 1,000$ & $\begin{array}{l}\text { Cell Signaling Technology, USA, } \\
\text { cat. no. } 4695\end{array}$ \\
\hline $\begin{array}{l}\text { Phospho-p38 (Thr180/Tyr182), } \\
\text { human }\end{array}$ & Rabbit, monoclonal & - & $1: 1,000$ & $\begin{array}{l}\text { Cell Signaling Technology, USA, } \\
\text { cat. no. } 4511\end{array}$ \\
\hline p38 MAPK, human & Rabbit, monoclonal & - & $1: 1,000$ & $\begin{array}{l}\text { Cell Signaling Technology, USA, } \\
\text { cat. no. } 8690\end{array}$ \\
\hline
\end{tabular}

Table III. The secondary antibodies and counterstaining of nuclei.

\begin{tabular}{|c|c|c|c|c|}
\hline Secondary detection system used & Host & Method & Dilution & Supplier \\
\hline Anti-mouse-IgG (H+L)-HRP & Goat & WB & $1: 10,000$ & Sungene Biotech, China, cat. no. LK2003 \\
\hline Anti-rabbit-IgG $(\mathrm{H}+\mathrm{L})-\mathrm{HRP}$ & Goat & WB & $1: 5,000$ & Sungene Biotech, China, cat. no. LK2001 \\
\hline $\begin{array}{l}\text { Anti-rabbit } \operatorname{IgG}(\mathrm{H}+\mathrm{L}), \mathrm{F}\left(\mathrm{ab}{ }^{\prime}\right) 2 \text { fragment } \\
\text { (Alexa Fluor }{ }^{\circledR} 488 \text { Conjugate) }\end{array}$ & Goat & IF & $1: 50$ & Cell Signaling Technology, USA, cat. no. 4412 \\
\hline $\begin{array}{l}\text { Hoechst } 33342(1 \mathrm{mg} / \mathrm{ml}) \text { nucleic } \\
\text { acid staining (DAPI) }\end{array}$ & - & IF & $1: 750$ & $\begin{array}{l}\text { Molecular Probes/Invitrogen, Carlsbad, } \\
\text { CA, USA, cat. no. A11007 }\end{array}$ \\
\hline
\end{tabular}



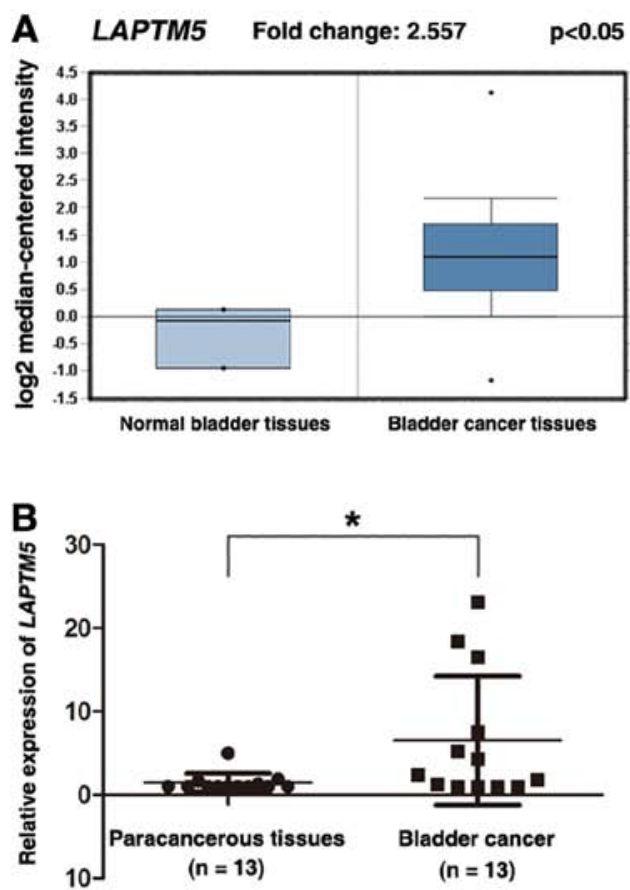

C a

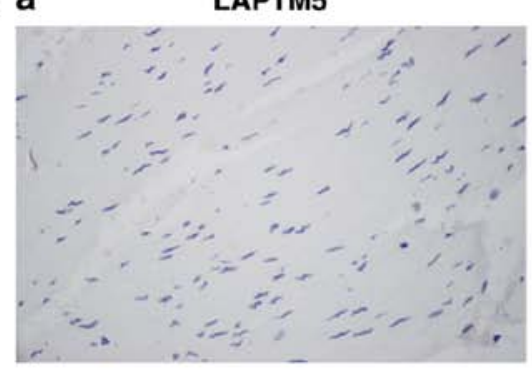

Bladder paracancerous tissue

c

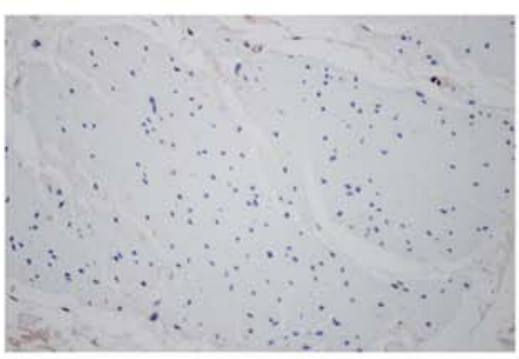

Bladder paracancerous tissue b

LAPTM5

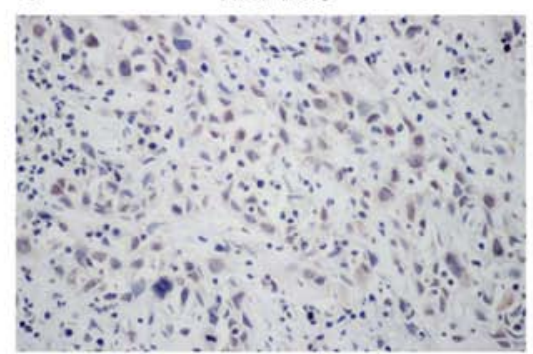

Bladder cancer tissue

d

LAPTM5

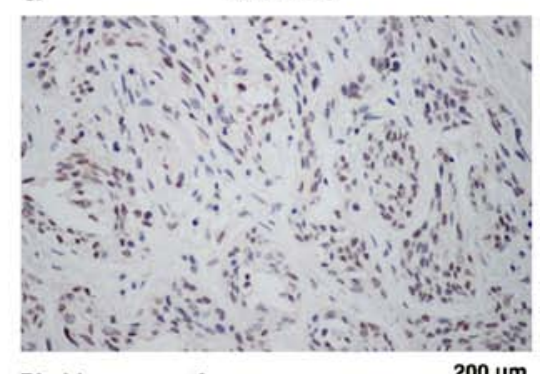

Bladder cancer tissue

$\underline{200 \mu \mathrm{m}}$

Figure 1. LAPTM5 is strongly upregulated in BCa tissues compared with paracancerous tissues and normal bladder tissues. (A) Upregulation of LAPTM5 mRNA expression in bladder cancer analyzed by Oncomine database. Analysis using the Oncomine database (www.oncomine.org) revealed increased LAPTM5 at transcriptional level in bladder cancer versus normal bladder tissues. (B) qRT-PCR analysis showed that the gene expression of LAPTM5 in bladder cancer tissues was significantly higher than the matched paracancerous tissues. The $G A P D H$ value was used as an internal control. " $p<0.05$. (C) Representative immunohistochemistry staining of LAPTM5 (brown) in (b and d) bladder cancer tissues and (a and c) paracancerous tissues. The scale bar for (C) is $200 \mu \mathrm{m}$.

A

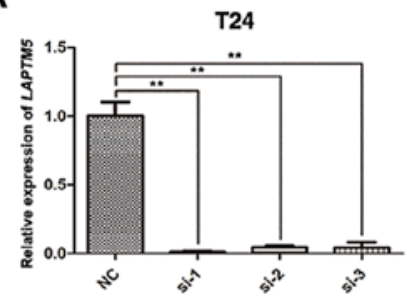

B

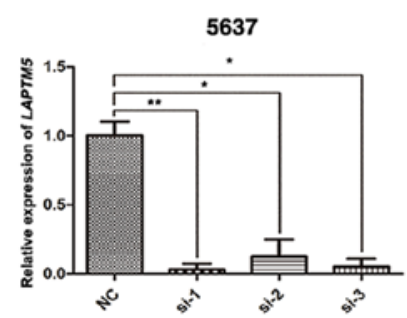

C

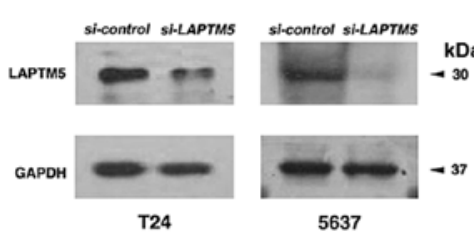

D a LAPTM5+DAPI

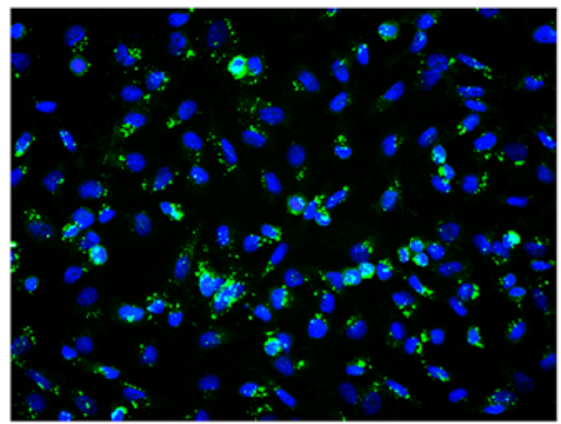

T24 si-control

c

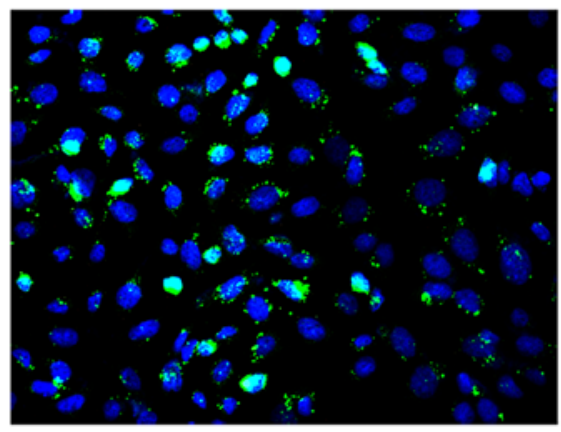

b LAPTM5+DAPI

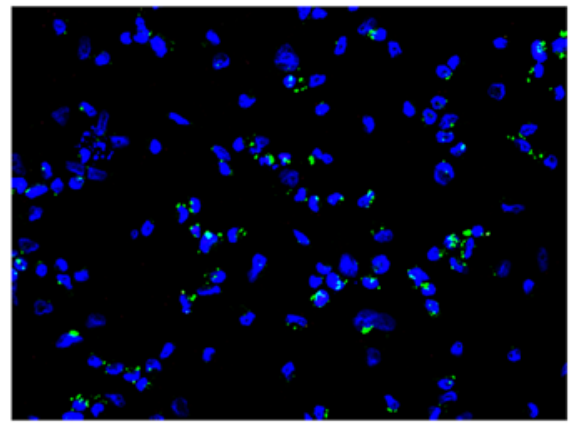

T24 si-LAPTM5

d LAPTM5+DAPI

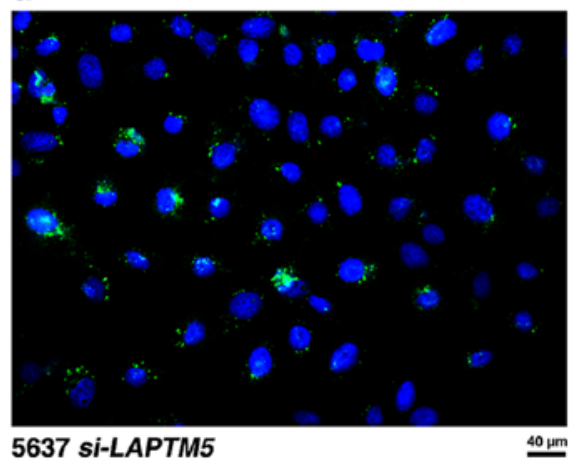

Figure 2. Establishment of BCa cell model with downregulated LAPTM5. (A and B) qRT-PCR validated the efficiency by using distinct siRNA to knockdown LAPTM5 at transcriptional level in the BCa cells T24 and 5637. All values shown are mean \pm SD of triplicate measurements and repeated three times with similar results, ${ }^{*} \mathrm{p}<0.05$ and ${ }^{* *} \mathrm{p}<0.01$. (C) Western blot analysis revealed a strongly decreased protein abundance of LAPTM5 by the LAPTM5-target-specific-siRNA (si-LAPTM5) treatment, comparing with control-siRNA (si-control) treatment. GAPDH was used as a loading control. (D) Representative immunofluorescence staining of LAPTM5 (green) in the BCa cells after si-LAPTM5 (b and d), compared with si-control (a and c) treatment. Nuclei were stained by DAPI (blue). The scale bar for (D) is $40 \mu \mathrm{m}$. 
A

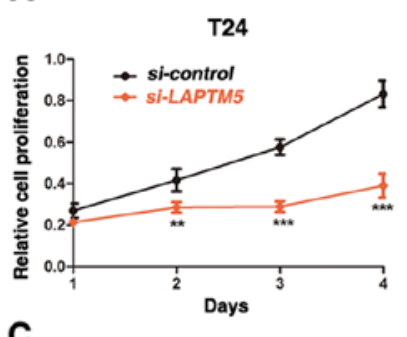

C
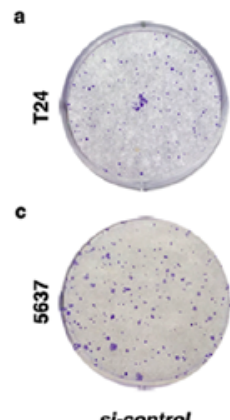

b

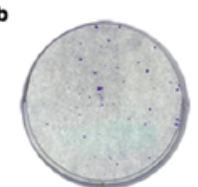

d

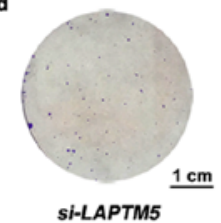

B

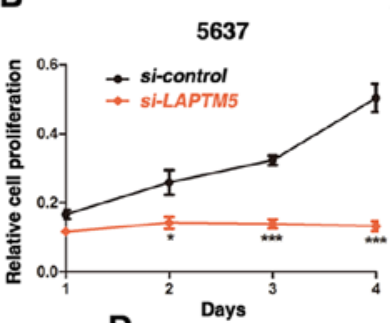

D

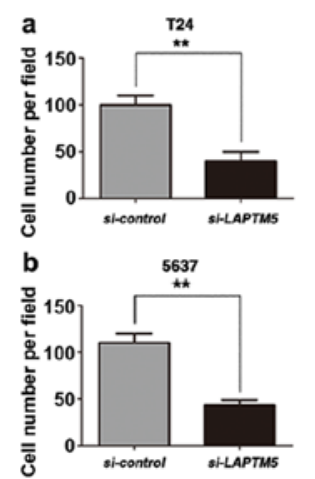

E

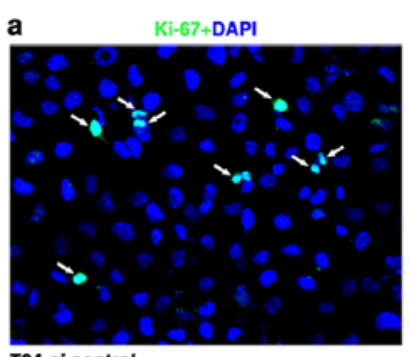

T24 si-control

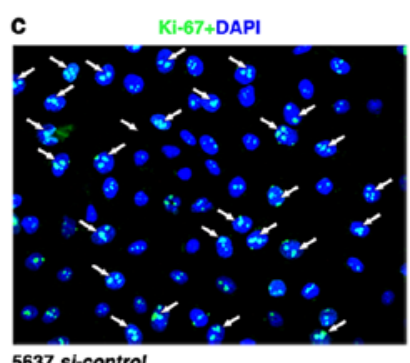

5637 si-control

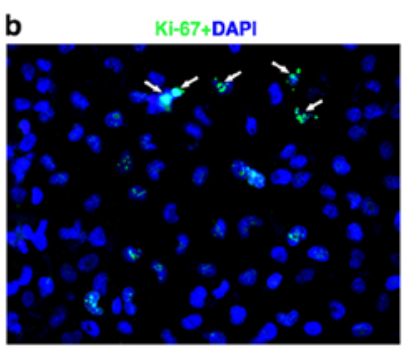

T24 si-LAPTM5

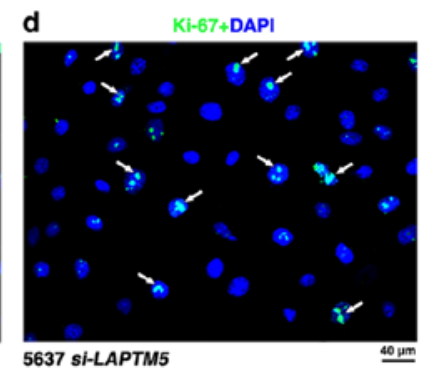

Figure 3. Downregulation of LAPTM5 represses BCa cell proliferation. (A and B) MTT assay was used to detect the viability of the two BCa cells treated by control-siRNA (si-control, black line) and LAPTM5-target-specific-siRNA (si-LAPTM5, red line). All values shown are mean \pm SD of triplicate measurements and repeated three times with similar results, ${ }^{* *} \mathrm{p}<0.01$ and ${ }^{* * *} \mathrm{p}<0.001$. (C) Alteration of cell survival for T24 (a and b) and 5637 (c and d) were analyzed by clonogenic survival assay after treatment with si-control and si-LAPTM5. (D) Clone number in each well was counted and statistically analyzed in the clonogenic survival assay. ${ }^{* *} \mathrm{p}<0.01$. (E) Cell proliferation of BCa cells treated by si-control (a and c) and si-LAPTM5 (b and d) was detected by Ki-67 staining (green), pointed by the arrows. Nuclei were stained by DAPI (blue). The scale bar for (C) is $1 \mathrm{~cm}$ and for (E) is $40 \mu \mathrm{m}$.

by MTT assay, suggesting that knockdown of LAPTM5 restrained $\mathrm{BCa}$ cells proliferation drastically (Fig. 3A and B). Clonogenic survival assay revealed a significant reduction for the colony forming efficiency in the LAPTM5-siRNAtreated BCa cells T24 and 5637, compared with the si-control group (Fig. 3C and D). Moreover, immunofluorescence staining showed that the LAPTM5-siRNA group exhibited considerably less Ki-67 positive cells than the si-control group (Fig. 3E).

Reduced LAPTM5 triggers cell cycle arrest at G0/G1 phase, but shows no significant changes on apoptosis in the BCa cells. Flow cytometry analysis was conducted to evaluate the effect of LAPTM5 knockdown on cell cycle in T24 and 5637 cells (Fig. 4A), indicating a significant cell cycle arrest at G0/G1 phase (Fig. 4B). Western blot analysis revealed that proteins involved in $\mathrm{G} 0 / \mathrm{G} 1$ phase regulation were strongly reduced (cyclin A1/2, cyclin D1 and CDK2/4) after LAPMT5-siRNA treatment (Fig. 4C). However, knockdown of LAPTM5 could not affect apoptosis in BCa cells significantly (Fig. 4D and E), as revealed by flow cytometry analysis.

Downregulation of LAPTM5 inhibits migration and invasion of $B C$ a cells. Transwell migration and invasion assay suggested that knockdown of LAPTM5 in BCa cells could reduce cell migration and invasion (Fig. 5A), which was confirmed by statistical analysis in Fig. 5B. Moreover, wound healing assay revealed that reduction of LAPTM5 in BCa cells could suppress the number of migrated cells (Fig. 5A). The gap closure (\%) was statistically analyzed (Fig. 5B).

Proteins involved in MAPK signaling pathway and EMT regulation are altered after LAPTM5 knockdown. Key members of the MAPK family including ERK1/2 and p38 were affected in the si-LAPTM5-treated T24 and 5637 cells (Fig. 5C). LAPTM5 knockdown strongly suppressed the expression of phosphorylated ERK1/2 (p-ERK1/2) and phosphorylated p38 (p-p38) in the $\mathrm{BCa}$ cells. In addition, proteins involved in the EMT process, including $\beta$-catenin, $\mathrm{N}$-cadherin, E-cadherin, claudin-1 and Slug, were analyzed by western blot analysis (Fig. 5D), showing that the epithelial marker E-cadherin was upregulated and mesenchymal marker N-cadherin, $\beta$-catenin, Slug, claudin-1 were downregulated after LAPTM5 knockdown.

\section{Discussion}

Our group has established a transcriptome analysis using bladder cancer tissues versus normal bladder tissues $(3,4)$. Among thousands of strongly altered genes involved in development of human bladder cancer (BCa), we selected the upregulated gene LAPTM5, which is in accordance with the result from the Oncomine database. LAPTM5 has been reported to be correlated with NEDD4 (8) which is upregulated in invasive $\mathrm{BCa}$ and could promote progression of $\mathrm{BCa}$ (9). Interestingly, our results showed that the expression of LAPTM5 was strongly enhanced in BCa tissues at both transcriptional and protein levels compared with paracancerous tissues. The LAPTM5-siRNA was used for LAPTM5 knockdown and the efficiency was confirmed by qRT-PCR, western blot analysis and immunofluorescence staining analyses. We observed that knockdown of LAPTM5 could reverse the EMT status, suggesting that deficiency of LAPTM5 could alleviate malignancy of BCa. Since several studies have reported that EMT was involved in cancer cell migration and invasion (16), we also observed that knockdown of LAPTM5 


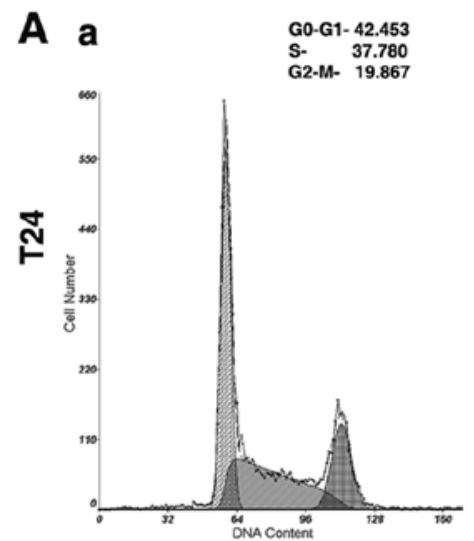

b
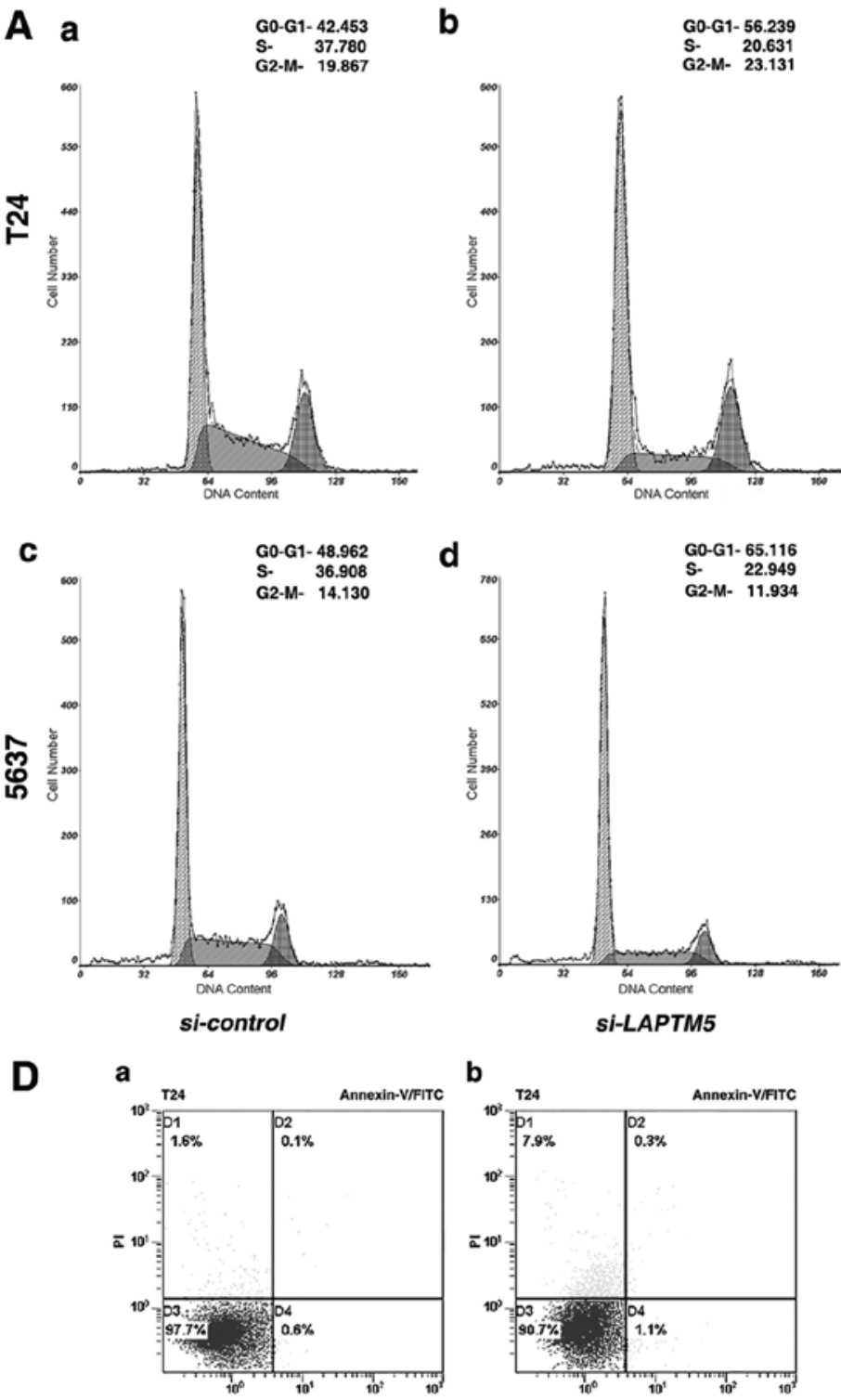

b

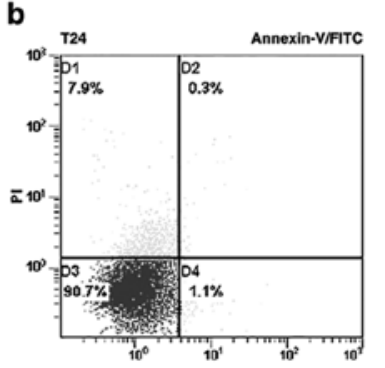

C

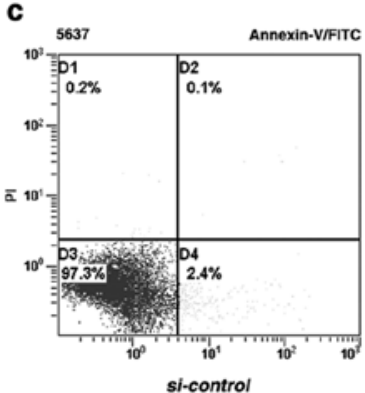

\section{d}

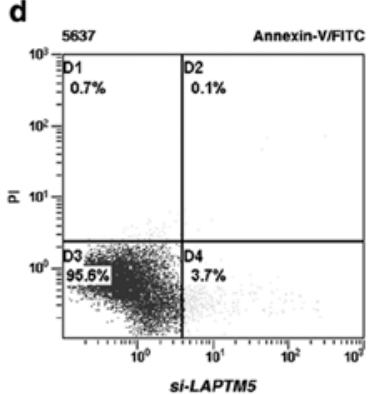

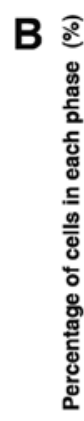

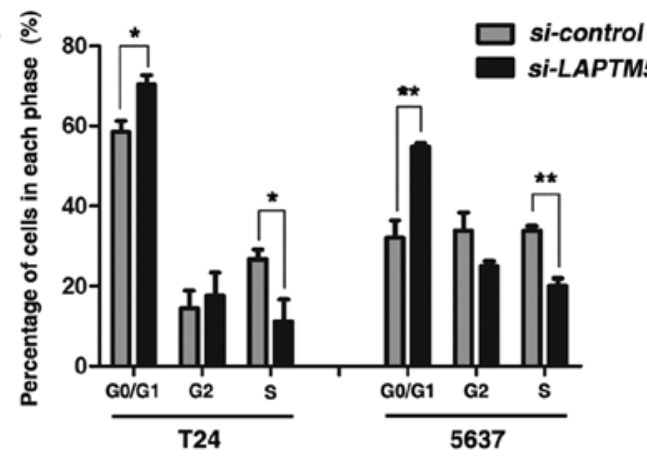

C

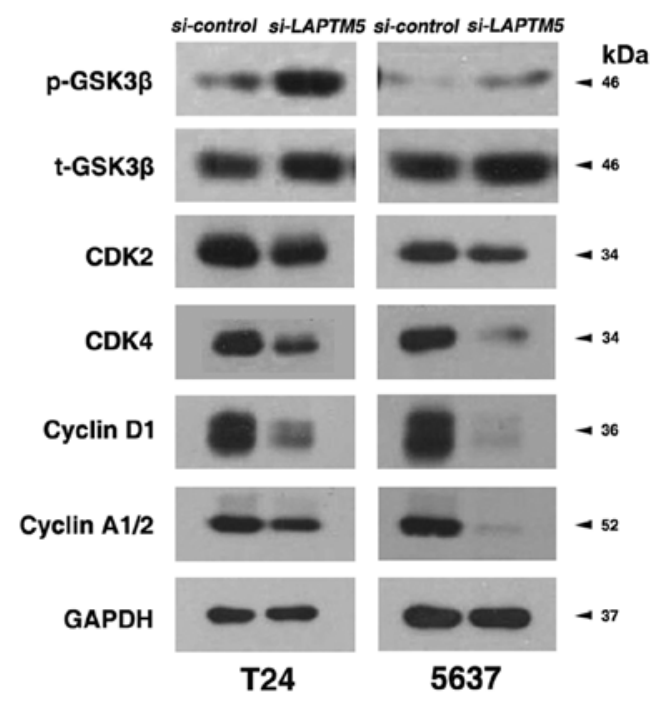

$\mathbf{E}$

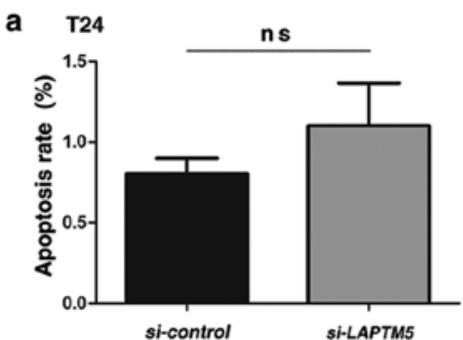

b

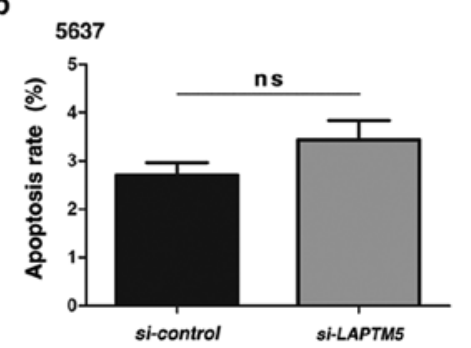

Figure 4. Knockdown of LAPTM5 induces cell cycle arrest at G0/G1 phase in BCa cells. (A) Flow cytometry analysis for T24 (a and b) and 5637 cells (c and d) treated with control-siRNA (si-control) (a and c) and LAPTM5-target-specific-siRNA (si-LAPTM5) (b and d) for $48 \mathrm{~h}$. (B) Alteration of percentages (\%) of cell populations at different stages of cell cycles in T24 and 5637 cells was statistically analyzed. All the values shown are mean \pm SD of triplicate measurements and repeated three times with similar results. " $\mathrm{p}<0.05$ and ${ }^{* * *} \mathrm{p}<0.01$. (C) Western blot analysis of proteins involved in the G0/G1 cell cycle regulation (cyclin A1/2, cyclin D1 and CDK2/4) and phosphorylated/total GSK-3 $\beta$ in the BCa cells. GAPDH abundance was used as a loading control. (D) Flow cytometry analysis of alterations of BCa cell apoptosis (T24 and 5637) by the transfection using si-control and si-LAPTM5. Calculation area of the apoptosis rate was percentage of Annexin $\mathrm{V}^{+} / \mathrm{PI}^{+}$cells. (E) Statistical analysis suggested no significant (ns) induction of apoptosis by the downregulation of LAPTM5 in human T24 (a) and 5637 (b) BCa cells.

suppressed migration and invasion of BCa cells. E-cadherin was found to be associated with epithelial cell migration and could play a key role in EMT progression (23), often marked by decreased E-cadherin and increased $\mathrm{N}$-cadherin (18).
After re-localization from membrane to cytoplasm and nucleus, $\beta$-catenin became a transcriptional coactivator to promote EMT (24). Slug is a zinc-finger transcription factor and has a functional role in triggering EMT (25), cancer 
A a
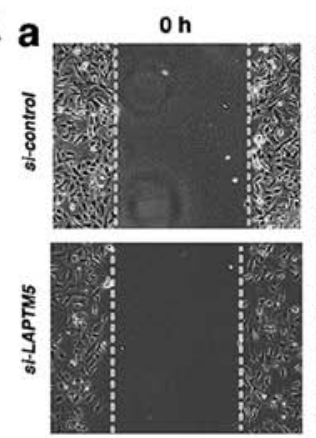

T24

C

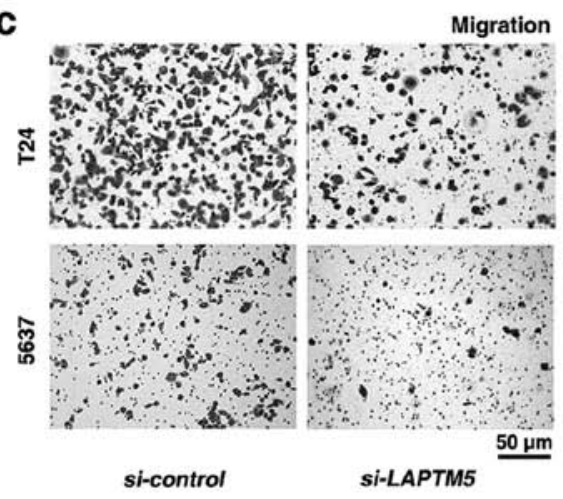

C si-control si-LAPTM5 si-control si-LAPTM5
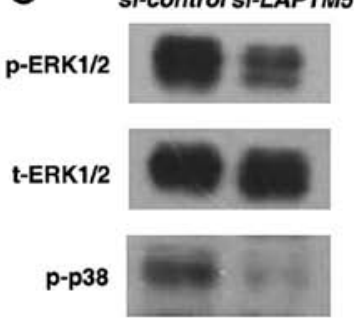

t-p38

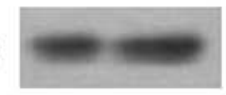

GAPDH

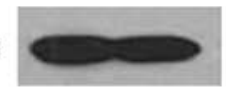

T24

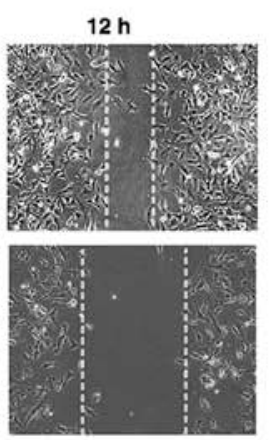
(1)
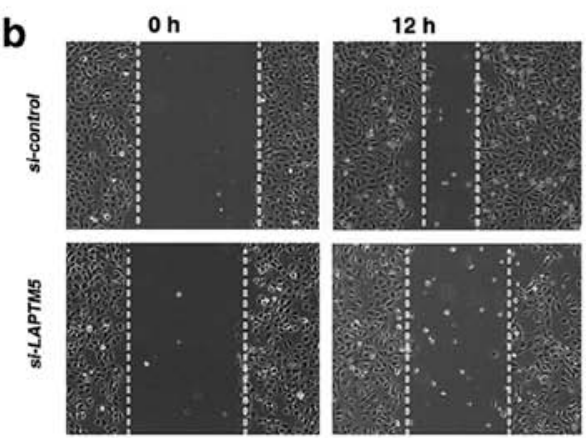

B a

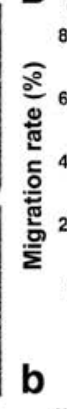

5637

d

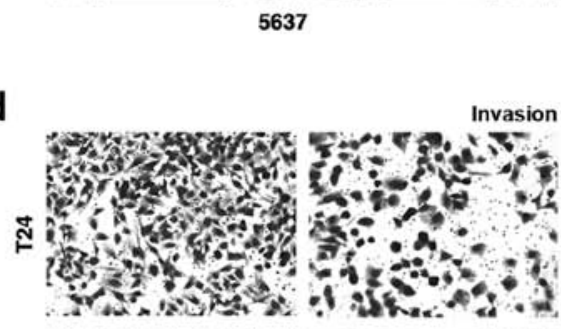

.

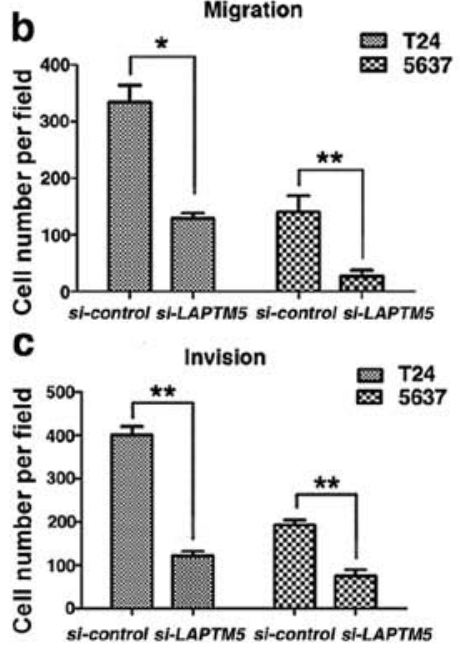

D si-control si-LAPTM5 si-control si-LAPTM5

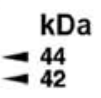

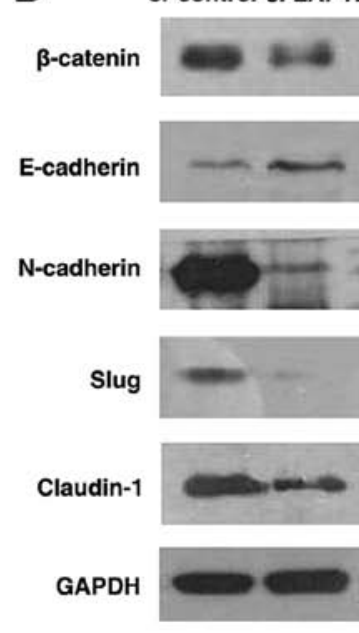

T24
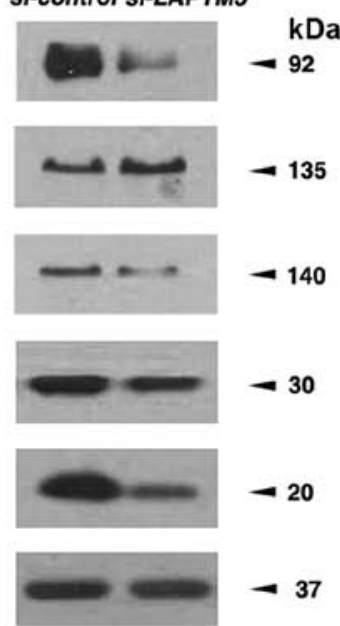

5637

Figure 5. Proteins involved in MAPK signaling pathway and EMT regulation are altered after LAPTM5 knockdown. (A) Wound healing assay (a and b), Transwell migration assay (c) and invasion assay (d) for T24 and 5637 treated with control-siRNA (si-control) and LAPTM5-target-specific-siRNA (si-LAPTM5). Scale bars for (c and d) are $50 \mu \mathrm{m}$. (B) Statistical analysis of wound healing assay (a), suggesting significantly reduced migration rate (\%) after siRNA treatment in T24 and 5637 cells. Statistical analysis of Transwell migration assay (b) and invasion assay (c), suggesting significantly reduced migrated cell number of T24 and 5637 cells after siRNA treatment. All values shown are mean \pm SD of triplicate measurements and repeated three times with similar results. ${ }^{*} \mathrm{p}<0.05$ and ${ }^{* *} \mathrm{p}<0.01$. (C) Western blot analysis of phosphorylated ERK1/2 (p-ERK1/2), total ERK1/2 (t-ERK1/2), phosphorylated p38 (p-p38) and total p38 (t-p38) in the T24 and 5637 cells by siRNA treatment. GAPDH was used as a loading control. (D) Western blot analysis for proteins involved in EMT regulation, revealing that $\mathrm{E}$-cadherin was strongly increased. In contrast, $\mathrm{N}$-cadherin, $\beta$-catenin, slug and claudin-1 were considerably decreased after siRNA treatment. GAPDH abundance was used as a loading control.

progression (26), invasion and migration (27). Consistently, our results showed that LAPTM5 knockdown resulted in an increase of E-cadherin and decrease of $\mathrm{N}$-cadherin, $\beta$-catenin and Slug in BCa cells (Fig. 5).

Another important phenomenon observed was that reduced LAPTM5 exhibited a negative effect on cell proliferation. Since cell proliferation was influenced by cell cycle and apop- tosis, we found that $\mathrm{BCa}$ cells lacking LAPTM5 significantly induced G0/G1 cell cycle arrest, but apoptotic rate of BCa cells showed no significant alteration. Proteins involved in cell cycle regulation, such as cyclin A1/2, cyclin D1 and CDK2/4, were inhibited by LAPTM5 knockdown.

Our microarray analysis also suggested that MAPK signaling pathway was linked with bladder cancer through 
regulating the cell cycle (3). Recent study reported that the LAPTM5 protein is a positive regulator of MAPK signaling pathway in macrophages (19). Similarly, our study also showed that p-ERK1/2 and p-p38 play important roles in regulating cell proliferation, survival and apoptosis (28) via connecting extracellular stimuli from cell membrane to nucleus were substantially downregulated after LAPTM5 knockdown. It is known that MAPK family members participate in regulating cell cycle in various manner (29). ERK mainly promotes progression of $\mathrm{G} 0 / \mathrm{G} 1$ to $\mathrm{S}$ phase and $\mathrm{p} 38$ primarily regulates G2 checkpoint $(30,31)$. Meloche and Pouysségur reported that activation of ERK1/2 could regulate the progression of G1 to $S$ phase by targeting cyclin D1 (29). Our results also revealed that LAPMT5 knockdown induced cell cycle arrest, which was confirmed by downregulation of related protein (cyclin D1 and CDK2/4) and upregulation of their upstream proteins p-GSK$3 \beta / \mathrm{t}-\mathrm{GSK} 3 \beta$ (Fig. 4). The above results suggested that cell cycle arrest induced by LAPTM5 knockdown may have a connection with MAPK signaling pathway in bladder cancer.

In conclusion, our results are the first to reveal that downregulation of LAPTM5 inhibited migration and invasion by suppressing EMT markers and reduced proliferation in $\mathrm{BCa}$ cells. Moreover, this process may be partially connected with the alteration of MAPK signal pathway.

\section{Acknowledgements}

The excellent technical assistance of Yuan Zhu, Shanshan Zhang and Danni Shan is gratefully acknowledged. This study was supported in part by grants from the Natural Sciences Foundation of Hubei Province (grant no. 2014CFA006), the Medical Science and Technology Project of Zhejiang Province (grant no. 2016KYB082) and the Fundamental Research Funds for the Central Universities (grant no. 2042015kf0153). The funders had no role in study design, data collection and analysis, decision to publish, or preparation of the manuscript.

\section{References}

1. Burger M, Catto JW, Dalbagni G, Grossman HB, Herr H, Karakiewicz P, Kassouf W, Kiemeney LA, La Vecchia C, Shariat S, et al: Epidemiology and risk factors of urothelial bladder cancer. Eur Urol 63: 234-241, 2013.

2. Rye PD, Nustad K and Stigbrand T: Tumor marker workshops. Tumour Biol 24: 165-171, 2003.

3. Cao R, Meng Z, Liu T, Wang G, Qian G, Cao T, Guan X, Dan H, Xiao Y and Wang X: Decreased TRPM7 inhibits activities and induces apoptosis of bladder cancer cells via ERK1/2 pathway. Oncotarget: Sep 20, 2016 (Epub ahead of print).

4. Wang G, Cao R, Wang Y, Qian G, Dan HC, Jiang W, Ju L, Wu M, Xiao Y and Wang X: Simvastatin induces cell cycle arrest and inhibits proliferation of bladder cancer cells via PPAR $\gamma$ signalling pathway. Sci Rep 6: 35783, 2016.

5. Ouchida R, Yamasaki S, Hikida M, Masuda K, Kawamura K, Wada A, Mochizuki S, Tagawa M, Sakamoto A, Hatano M, et al A lysosomal protein negatively regulates surface $\mathrm{T}$ cell antigen receptor expression by promoting CD3zeta-chain degradation. Immunity 29: 33-43, 2008.

6. Seimiya M, O-Wang J, Bahar R, Kawamura K, Wang Y, Saisho H and Tagawa M: Stage-specific expression of Clast6/E3/LAPTM5 during $\mathrm{B}$ cell differentiation: Elevated expression in human B lymphomas. Int J Oncol 22: 301-304, 2003.

7. Adra CN, Zhu S, Ko JL, Guillemot JC, Cuervo AM, Kobayashi H, Horiuchi T, Lelias JM, Rowley JD and Lim B: LAPTM5: A novel lysosomal-associated multispanning membrane protein preferentially expressed in hematopoietic cells. Genomics 35: 328-337, 1996
8. Pak Y, Glowacka WK, Bruce MC, Pham N and Rotin D: Transport of LAPTM5 to lysosomes requires association with the ubiquitin ligase Nedd4, but not LAPTM5 ubiquitination. J Cell Biol 175: 631-645, 2006.

9. Ingham RJ, Gish G and Pawson T: The Nedd4 family of E3 ubiquitin ligases: Functional diversity within a common modular architecture. Oncogene 23: 1972-1984, 2004.

10. Wang X, Trotman LC, Koppie T, Alimonti A, Chen Z, Gao Z, Wang J, Erdjument-Bromage H, Tempst P, Cordon-Cardo C, et al: NEDD4-1 is a proto-oncogenic ubiquitin ligase for PTEN. Cell 128: 129-139, 2007.

11. Shao GZ, Zhou RL, Zhang QY, Zhang Y, Liu JJ, Rui JA, Wei $X$ and Ye DX: Molecular cloning and characterization of LAPTM4B, a novel gene upregulated in hepatocellular carcinoma. Oncogene 22: 5060-5069, 2003.

12. Zhang H, Wei Q, Liu R, Qi S, Liang P, Qi C, Wang A, Sheng B, Li L and Xu Y: Overexpression of LAPTM4B-35: A novel marker of poor prognosis of prostate cancer. PLoS One 9: e91069, 2014.

13. Xiao M, Jia S, Wang H, Wang J, Huang Y and Li Z: Overexpression of LAPTM4B: An independent prognostic marker in breast cancer. J Cancer Res Clin Oncol 139: 661-667, 2013.

14. Huber MA, Kraut N and Beug H: Molecular requirements for epithelial-mesenchymal transition during tumor progression. Curr Opin Cell Biol 17: 548-558, 2005.

15. Nieto MA: Epithelial plasticity: A common theme in embryonic and cancer cells. Science 342: 1234850, 2013.

16. Yang $\mathrm{J}$ and Weinberg RA: Epithelial-mesenchymal transition: At the crossroads of development and tumor metastasis. Dev Cell 14: 818-829, 2008.

17. Wei SC, Fattet L and Yang J: The forces behind EMT and tumor metastasis. Cell Cycle 14: 2387-2388, 2015.

18. Yilmaz $\mathrm{M}$ and Christofori G: EMT, the cytoskeleton, and cancer cell invasion. Cancer Metastasis Rev 28: 15-33, 2009.

19. Glowacka WK, Alberts P, Ouchida R, Wang JY and Rotin D: LAPTM5 protein is a positive regulator of proinflammatory signaling pathways in macrophages. J Biol Chem 287: 27691-27702, 2012.

20. Park JS, Kwon JK, Kim HR, Kim HJ, Kim BS and Jung JY: Farnesol induces apoptosis of DU145 prostate cancer cells through the PI3K/ Akt and MAPK pathways. Int J Mol Med 33: 1169-1176, 2014.

21. Gupta J, Igea A, Papaioannou M, Lopez-Casas PP, Llonch E, Hidalgo M, Gorgoulis VG and Nebreda AR: Pharmacological inhibition of $\mathrm{p} 38$ MAPK reduces tumor growth in patient-derived xenografts from colon tumors. Oncotarget 6: 8539-8551, 2015.

22. Livak KJ and Schmittgen TD: Analysis of relative gene expression data using real-time quantitative PCR and the 2(-Delta Delta C(T)) method. Methods 25: 402-408, 2001.

23. Wheelock MJ and Johnson KR: Cadherins as modulators of cellular phenotype. Annu Rev Cell Dev Biol 19: 207-235, 2003.

24. Harris TJ and Peifer M: Decisions, decisions: Beta-catenin chooses between adhesion and transcription. Trends Cell Biol 15: 234-237, 2005.

25. Bolós V, Peinado H, Pérez-Moreno MA, Fraga MF, Esteller M and Cano A: The transcription factor Slug represses E-cadherin expression and induces epithelial to mesenchymal transitions: A comparison with Snail and E47 repressors. J Cell Sci 116: 499-511, 2003.

26. Li Y, Wu Y, Abbatiello TC, Wu WL, Kim JR, Sarkissyan M, Sarkissyan S, Chung SS, Elshimali Y and Vadgama JV: Slug contributes to cancer progression by direct regulation of ERa signaling pathway. Int J Oncol 46: 1461-1472, 2015.

27. Sun Y, Song GD, Sun N, Chen JQ and Yang SS: Slug overexpression induces stemness and promotes hepatocellular carcinoma cell invasion and metastasis. Oncol Lett 7: 1936-1940, 2014.

28. Xiao Y, Karnati S, Qian G, Nenicu A, Fan W, Tchatalbachev S, Höland A, Hossain H, Guillou F, Lüers GH, et al: Cre-mediated stress affects sirtuin expression levels, peroxisome biogenesis and metabolism, antioxidant and proinflammatory signaling pathways. PLoS One 7: e41097, 2012.

29. Meloche S and Pouysségur J: The ERK1/2 mitogen-activated protein kinase pathway as a master regulator of the G1- to S-phase transition. Oncogene 26: 3227-3239, 2007.

30. MacCorkle RA and Tan TH: Mitogen-activated protein kinases in cell-cycle control. Cell Biochem Biophys 43: 451-461, 2005.

31. Tsai SC, Huang WW, Huang WC, Lu CC, Chiang JH, Peng SF Chung JG, Lin YH, Hsu YM, Amagaya S, et al: ERK-modulated intrinsic signaling and $\mathrm{G}(2) / \mathrm{M}$ phase arrest contribute to the induction of apoptotic death by allyl isothiocyanate in MDA-MB-468 human breast adenocarcinoma cells. Int J Oncol 41: 2065-2072, 2012. 